\title{
Miniature Imaging Device with a Portable Processing System for Fluorescence Detection of Nitric Oxide in Living Cells
}

\author{
Anek Wuthayavanich, Makito Haruta, ${ }^{*}$ Hiroaki Takehara, Toshihiko Noda, \\ Kiyotaka Sasagawa, Takashi Tokuda, and Jun Ohta \\ Nara Institute of Science and Technology, 8916-5 Takayama, Ikoma, Nara 630-0192, Japan
}

(Received May 2, 2016; accepted August 10, 2016)

Keywords: complementary metal-oxide semiconductor (CMOS) image sensor, portable processing system, fluorescence imaging, nitric oxide, cell culture experiment

Fluorescence imaging is a powerful tool for studying the functions of nitric oxide (NO). To improve this method of studying the mechanisms of NO related with behaviors in freely moving animals, we developed a miniature imaging device with a portable processing system. The miniature device was integrated with a complementary metal-oxide semiconductor (CMOS) image sensor, blue light-emitting diodes as an excitation NO probe, and an absorption filter for filtering the excitation light. The portable processing system is lightweight and can be placed on a small animal body. We successfully detected fluorescent images in living cells and captured mouse brain surfaces under implantation conditions using the imaging device. Furthermore, we determined that our device contributes to understanding the in vivo functions of NO in a small animal body.

\section{Introduction}

Nitric oxide (NO) was initially identified as a molecular chemical compound that controls vascular systems by relaxing smooth muscle cells around blood vessels. ${ }^{(1)}$ This finding strongly fostered research on its functions. Until now, NO has been reported to be involved in blood flow systems, immune responses, and neurotransmitters. ${ }^{(2)}$ The deficiency of NO is related to diseases such as diabetes mellitus, ${ }^{(3)}$ hypertension, ${ }^{(4)}$ and other chronic ailments. On the other hand, NO is also used as a medical treatment, such as for the treatment of newborn infants with hypoxemic respiratory failure(5) and to prevent ischemic stroke. ${ }^{(6)}$ Therefore, many techniques, such as chemiluminescence $^{(7)}$ and electron paramagnetic resonance (EPR) spectroscopy, ${ }^{(8)}$ have been developed. Although these methods can detect NO in the nM range, they cannot be applied to in vivo research owing to toxicity from chemical reactions and the large size of the equipment. An amperometric method was thus developed for the detection of low concentrations of NO in small animals; ${ }^{(9)}$ nevertheless, it is difficult to apply that method for observing NO in a small specific area, such as around the tiny blood vessels of a guinea pig. ${ }^{(10)}$

Among the many methods of NO detection, fluorescence imaging is appropriate for use in living organisms, including plants or small animals, owing to its high sensitivity, fast response time, and biocompatibility. In small animals, this method is widely used for NO detection. For example, Terpolilli et al. ${ }^{(6)}$ studied the functions of NO to prevent ischemic brain damage. However, it

"Corresponding author: e-mail: m-haruta@ms.naist.jp 
is difficult for use in the long-term detection of NO in animals because of the restriction of a microscope, which requires a fixed area and is bulky in size. In living cells, studying physiological factors associated with $\mathrm{NO}$ by fluorescence microscopy requires dynamic focusing and a wide area. This is not convenient for tracking the mechanisms of NO.

Therefore, for NO detection in both living cells and freely moving animals, we developed a tiny imaging device and a portable visualization system for NO detection in human umbilical vein endothelial cells (HUVECs). We show that our device is suitable for fluorescence detection in living cells by directly culturing cells on the sensor and applying our system to the detection of calcein acetoxymethyl ester (calcein AM) fluorescent dye, which is used for staining HUVECs. Calcein AM has the same excitation and emission wavelengths as a NO fluorescent probe. Next, we demonstrate that our imaging device can be applied to the fluorescence imaging of diaminofluorophore 4-amino-5-methylamino-2'-7'-difluorofluorescein diacetate (DAF-FM DA), which is used for NO detection in HUVECs. The overview of this study is shown in Fig. 1 and is described as follows. First, HUVECs were stained with an NO probe (DAF- FM DA) and subjected to fluorescence using a $455-490 \mathrm{~nm}$ light source. Then, the image sensor captured the fluorescence of the NO probe and transmitted the image signal to the portable processing system. From that point, the processing system processed the signal from the image sensor and transferred the digital data to a computer via a universal serial bus (USB) port. Moreover, we confirm that our system can be applied to brain imaging with a freely moving animal. Our successful in vitro results lead to the detection of NO inside an animal's body, which is related to certain diseases and behaviors.

\section{Materials and Methods}

\subsection{Specifications of the complementary metal-oxide semiconductor (CMOS) image sensor chip}

The CMOS image sensor was designed in our laboratory to achieve a small size $(320 \times 1025$ $\left.\mu \mathrm{m}^{2}\right)$. It is operated by four pins ( $\left.\mathrm{V}_{\mathrm{DD}}, \mathrm{GND}, \mathrm{CLK}, \mathrm{V}_{\mathrm{OUT}}\right)$. The specifications of the image sensor are shown in Table 1. The relationship of the light sensitivity and the voltage output of the sensor is $9 \mathrm{mV} /\left(\mathrm{nW} / \mathrm{mm}^{2}\right)$ and is shown in Fig. 2.

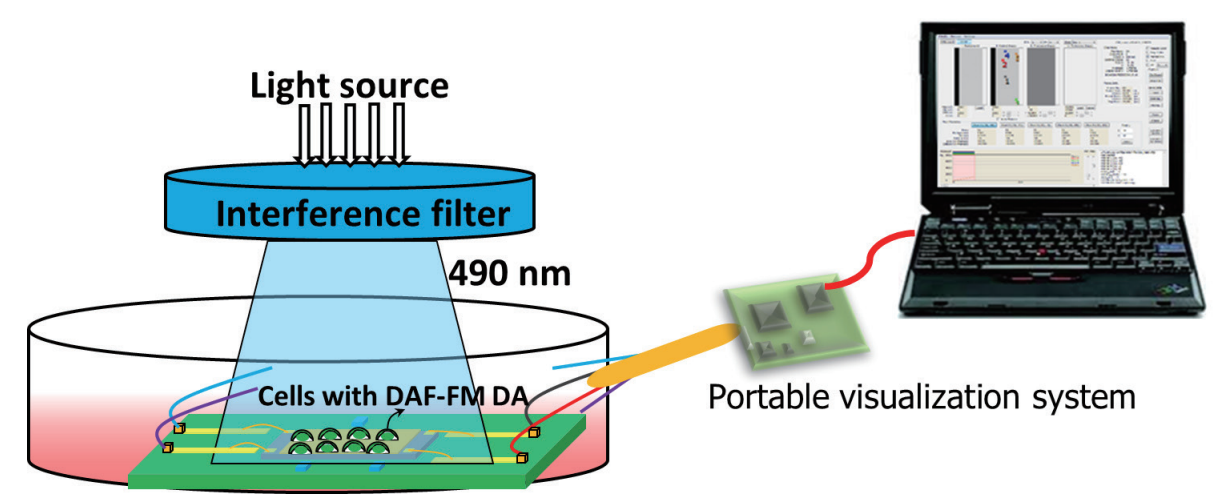

Fig. 1. (Color online) Overview of a miniature imaging device and a portable visualization system for NO detection in HUVECs. 
Table 1

CMOS image sensor chip specifications.

\begin{tabular}{lc}
\hline Process technology & $0.35 \mu \mathrm{m}$ 2-poly 4-metal standard CMOS process \\
Pixel structure & 3-transistor active pixel sensor \\
Photodiode type & Nwell_Psubstrate junction \\
Pixel size & $7.5 \times 7.5 \mu \mathrm{m}^{2}$ \\
Pixel number & $30 \times 90,40 \times 90$ \\
Fill factor & $35 \%$ \\
Dimension & $320 \times 1025 \times 350 \mu \mathrm{m}^{3}$ \\
Supply voltage & $3.3 \mathrm{~V}$ \\
Output voltage & $0.35-1.3 \mathrm{~V}$ \\
\hline
\end{tabular}

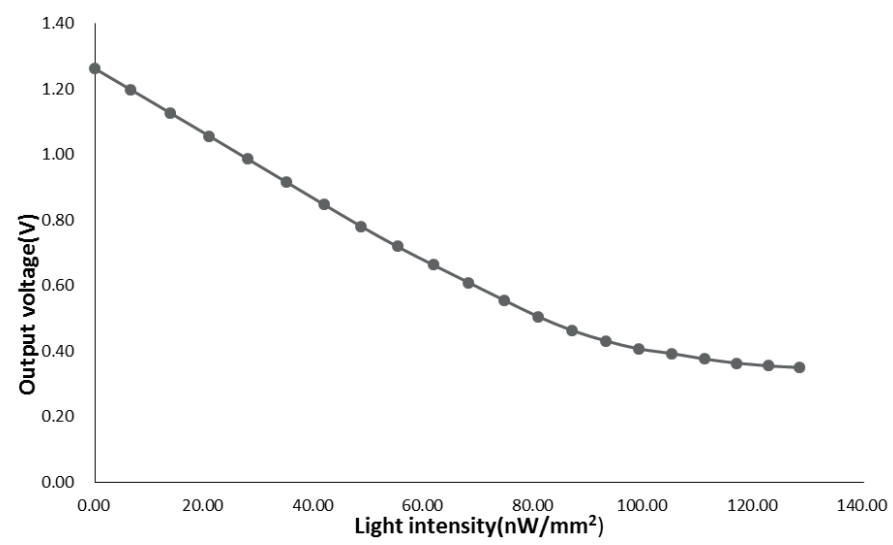

Fig. 2. Output voltage of an image sensor as a function of green light intensity.

\subsection{Fabrication of miniature imaging device}

Our imaging device is composed of the CMOS image sensor $(30 \times 90$ pixels $)$, blue lightemitting diodes (LEDs) (EPISTAR ES-CEGHM12A) for the excitation of an NO fluorescence probe, and an absorption filter ( $8-10 \mu \mathrm{m}$ thickness) attached to the image sensor for the rejection of excitation light. The fabrication of this filter was reported in our previous research. ${ }^{(11)}$

The device fabrication is described in Fig. 3. First, the image sensor and three blue LEDs were attached to a printed circuit board (PCB) using an epoxy resin and then baked on a hotplate at 120 ${ }^{\circ} \mathrm{C}$ for 15 min [Fig. 3(a)]. Second, the image sensor and LEDs were electrically connected to the PCB pads by using a wire bonding machine $(7700 \mathrm{CP}$, West Bond, Inc.). They were then covered with an epoxy resin to increase the durability [Fig. 3(b)]. Third, the film of the absorption filter was placed on the image sensor surface and baked at $120^{\circ} \mathrm{C}$ for $3 \mathrm{~h}$ in a vacuum oven to strengthen the attachment between the filter and the image sensor [Fig. 3(c)]. A photograph of the imaging device is shown in Fig. 3(d).

\subsection{Creating a small chamber for cell culture on the imaging device}

To perform cell culture on the imaging device, a small chamber, made of acrylic material, was attached to the PCB with the imaging device using an epoxy resin (Fig. 4). Finally, the chamber and the imaging device were coated with a Parylene film (1.5 $\mu \mathrm{m}$ thickness) by an evaporation method. 


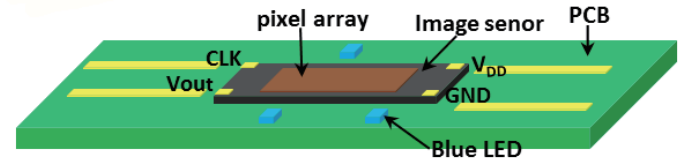

(a)

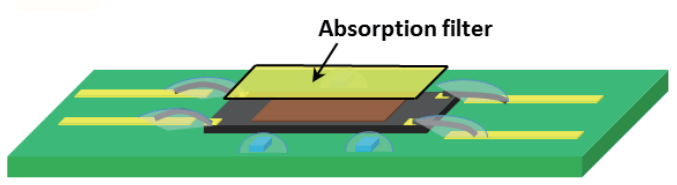

(c)

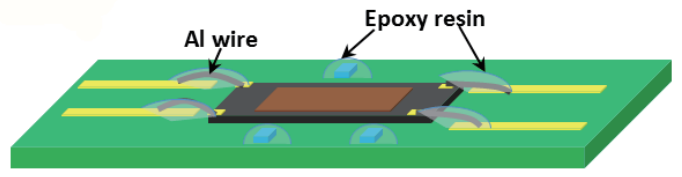

(b)

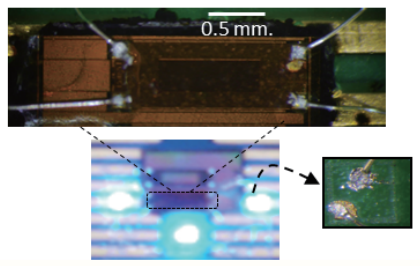

(d)

Fig. 3. (Color online) (a)-(c) Fabrication of the miniature imaging device. (d) Photograph of the fabricated imaging device.

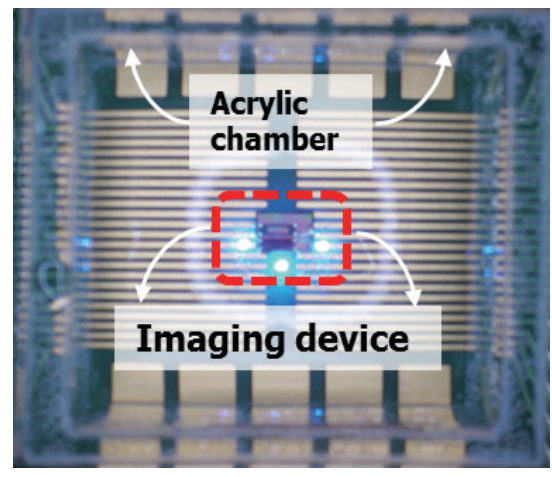

Fig. 4. (Color online) Photograph of the small chamber with the imaging device and LEDs.

\subsection{Portable processing system}

The small $\left(5.2 \times 2.8 \mathrm{~cm}^{2}\right)$ and lightweight $(\sim 8 \mathrm{~g})$ processing system was designed in our laboratory for managing the signal from the image sensor and transferring the image data to a computer. The specifications of the portable system are shown in Table 2. Its operation, as outlined in Fig. 5(a), is described below.

First, a microcontroller generates a clock signal to the image sensor for scanning each pixel. The image sensor then immediately sends the voltage signal of each pixel that depends on light intensity to the microcontroller. These analog signals are converted to digital signals by a 12-bit analog-to-digital converter (ADC) module, which is built into the microcontroller. Afterwards, these digital signals are sent to a computer via the USB interface. Finally, all of the digital signals are managed for displaying the image using Visual $\mathrm{C}++$ software developed in our laboratory. The feature functions of this program were designed for analyzing fluorescence imaging and saving the data in a storage system. A photograph of the portable processing system is shown in Fig. 5(c). Our processing system changes the exposure time of the imaging device from 25 to $200 \mathrm{~ms}$. 
Table 2

Control board specifications.

\begin{tabular}{lc}
\hline Microcontroller & DSPIC33EP256MU806 \\
ADC resolution & $12 \mathrm{bit}$ \\
Reference voltage of ADC & $2.65 \mathrm{~V}$ \\
Maximum frame rate & $25 \mathrm{fps}$ \\
Interface & USB 2.0 full speed \\
Dimension & $5.2 \times 2.8 \mathrm{~cm}^{2}$ \\
Weight & $\sim 8 \mathrm{~g}$ \\
\hline
\end{tabular}
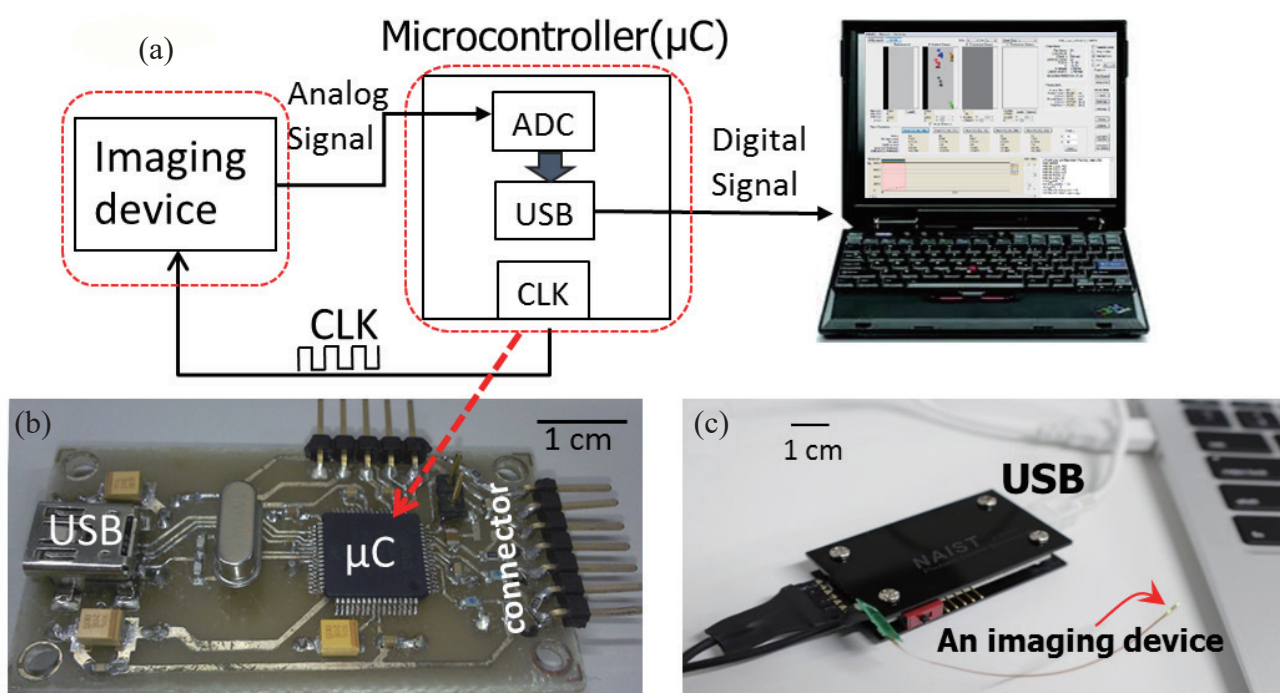

Fig. 5. (Color online) Portable image processing system. (a) Block diagram of the interface between the image sensor and computer via the USB port. (b) Photograph of the control board. (c) Photograph of the portable processing system connected with a flexible imaging device.

\subsection{Cell culture of human umbilical vein endothelial cells}

The HUVECs, which were purchased from PromoCell, were cultured in a petri dish containing $10 \mathrm{ml}$ of medium and serum mixed with growth factors. The cells were incubated in an incubator at $37{ }^{\circ} \mathrm{C}$ with $5 \% \mathrm{CO}_{2}$.

\subsection{Fluorescence imaging}

The fluorescent dyes used in this study for fluorescence imaging are (1) calcein, $2.5 \mu \mathrm{M}$ in PBS and (2) DAF-FM DA, $5 \mu \mathrm{M}$ in DMSO (Sigma-Aldrich Co.). To validate the imaging device for the detection of the fluorescence signal, we stained the HUVECs with calcein AM and measured the fluorescence with the proposed device. The excitation and emission wavelengths of calcein AM were 495 and $515 \mathrm{~nm}$, respectively, which are the same as those of the fluorescent dye for NO detection (DAD-FM DA). 
Next, the device was used for NO detection in HUVECs by using a DAD-FM DA fluorescent dye. The fluorescence detection procedure is described as follows. First, HUVECs were stained with a fluorescent dye and incubated in the dark for $30 \mathrm{~min}$. After staining, these cells were poured on the imaging device and the cell sediment was awaited on the sensor surface. Finally, the 12 $\mathrm{mW} / \mathrm{cm}^{2}$ excitation light at wavelengths of $455-490 \mathrm{~nm}$ was illuminated and the green fluorescence was immediately captured by the imaging device. The exposure time of the imaging device was set to $25 \mathrm{~ms}$.

\subsection{Animal preparation}

A C57BL/6 mouse (SLC Co., Japan, 10 weeks of age, $25 \mathrm{~g}$ ) was used in this study. All animal procedures conformed to the animal care and experimentation guidelines of the Nara Institute of Science and Technology. Urethane anesthesia $(1.0 \mathrm{~g} / \mathrm{kg})$ was injected into the abdominal cavity of the mouse using a syringe. The skull of the mouse was exposed and cleaned, and the primary sensory cortex (posterior $0.5 \mathrm{~mm}$, lateral $2.5 \mathrm{~mm}$ ) was observed. A craniotomy (less than $10 \mathrm{~mm}$ square) was opened above the observed region. The imaging device was set on the region and fixed with dental cement.

\section{Results}

\subsection{Stability of the portable processing system}

We characterized the accuracy of our portable processing system while connecting it to the imaging device. The noise distribution from the imaging device with this system is shown in Fig. 6. It shows that the total noise from our system is $18 \mathrm{mV}$.

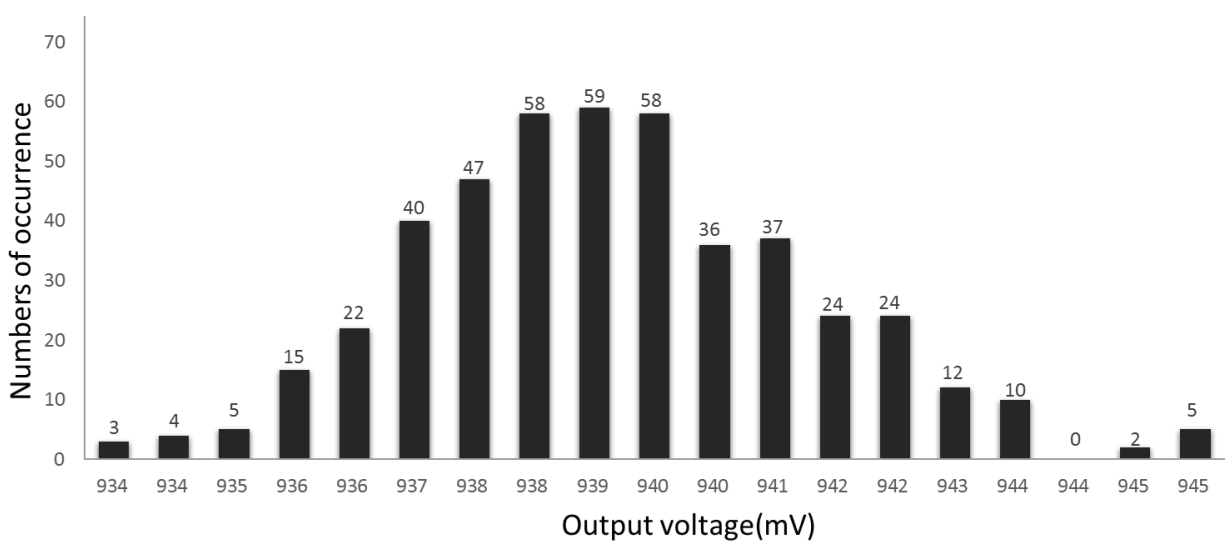

Fig. 6. Noise distribution of the imaging system. 


\subsection{Cell culture on small chamber with the imaging device}

To employ fluorescence imaging in an animal body, we verified the biocompatibility of the imaging device. The imaging device was coated with the Parylene film for waterproofing. We performed cell culture on the surface of the imaging device and checked the survival rate of the cultured cells. This study used HUVECs, which were stained with calcein AM dye. Figure 7 shows that the cells survived and proliferated to cover 80 to $90 \%$ of the surface of the imaging device.

\subsection{Florescence imaging with HUVECs on imaging device with portable processing system}

We investigated the sensitivity of the imaging device with the portable processing system for the detection of DAF-FM DA fluorescence, which was used for NO detection in the HUVECs. In this experiment, we used an external light source for the excitation of DAF-FM DA. As shown in Fig. 8(a), the fluorescence of DAF-FM DA emitted from HUVECs is shown using a microscope at points 1, 2, and 3. The exposure time of the microscopic fluorescent image was $2 \mathrm{~s}$. These fluorescent images are equivalent to the image from the imaging device shown in Fig. 8(b). Our imaging device with the portable processing system successfully detected fluorescence from HUVECs, which were stained by DAF-FM DA. In these fluorescence experiments, we used the same light source from the microscope. The intensity of the light source is $12 \mathrm{~mW} / \mathrm{cm}^{2}$.

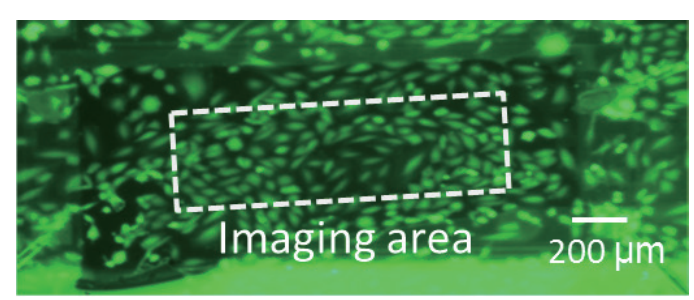

Fig. 7. (Color online) HUVECs stained by calcein AM dye survive on the surface of the imaging device.

(a) Microscope

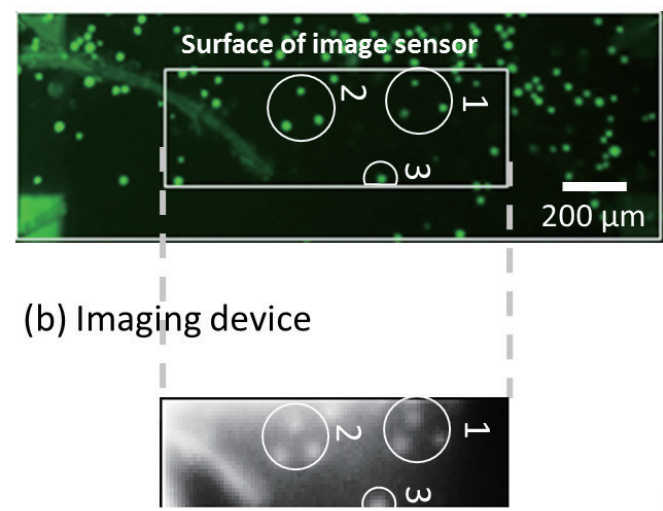

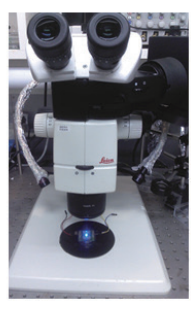

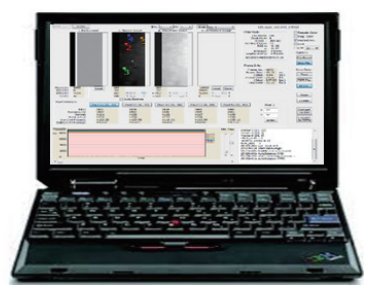

Fig. 8. (Color online) Detection of fluorescence of DAF-FM DA from HUVEC using the microscope and imaging device. 


\subsection{Brain surface imaging using imaging device with portable processing system}

We implanted the imaging device in the mouse head, as shown in Fig. 9(a), and attached the processing system on the mouse back, as shown in Fig. 9(b). Figure 9(d) shows the image of the brain surface that was captured by the imaging device at the dotted rectangular shape in Fig. 9(c). As shown 9(d), the imaging device clearly detected blood vessels on the brain surface.

\section{Discussion}

To accomplish our study on NO detection, we first had to ensure that our imaging device could be used for the direct culture of HUVECs. As shown in Fig. 7, HUVECs were attached, spread, and increased in number until the coverage reached approximately 80 to $90 \%$ on the imaging device. This result was mainly attributable to the property of Parylene coated on the device. Parylene has been reported as being a biocompatible material for tissues. ${ }^{(12)}$

Next, we determined the noise of our portable system for realizing the scope for fluorescence imaging. The noise of the system (Fig. 6) was caused by (1) an image sensor, such as short noise and thermal resistance noise, ${ }^{(13)}$ and (2) electric components, such as a microcontroller. To validate the effect from noise, the Rose criterion advises that the signal-to-noise ratio $(\mathrm{S} / \mathrm{N})$ should be greater than 5. (14) On the other hand, it can be deduced from Fig. 2 that the difference in fluorescence intensity from the background of our image sensor should be higher than $1.28 \mathrm{nW} / \mathrm{mm}^{2}$.

To verify the imaging device for fluorescence detection using the NO probe, we analyzed the ability of the filter to reject the excitation light and sensor sensitivity by detecting DAF-FM DA. In a comparison between the images captured by the microscope [Fig. 8(a)] and the image sensor [Fig. 8(b)], the fluorescence could only be observed in the area composed of cells. On the other hand, the filter could absorb excitation light and permit some green fluorescence to pass through the image sensor. The transmittance of DAF-FM DA fluorescence through the filter at the peak of emission $\left(515 \mathrm{~nm}\right.$ ) is only $10 \%,{ }^{(11)}$ however, with the sensitivity of our miniature imaging device (Fig. 2) and portable processing system (12 bit ADC), we detected the fluorescence from HUVECs stained by DAF-FM DA without the use of bulky optics.
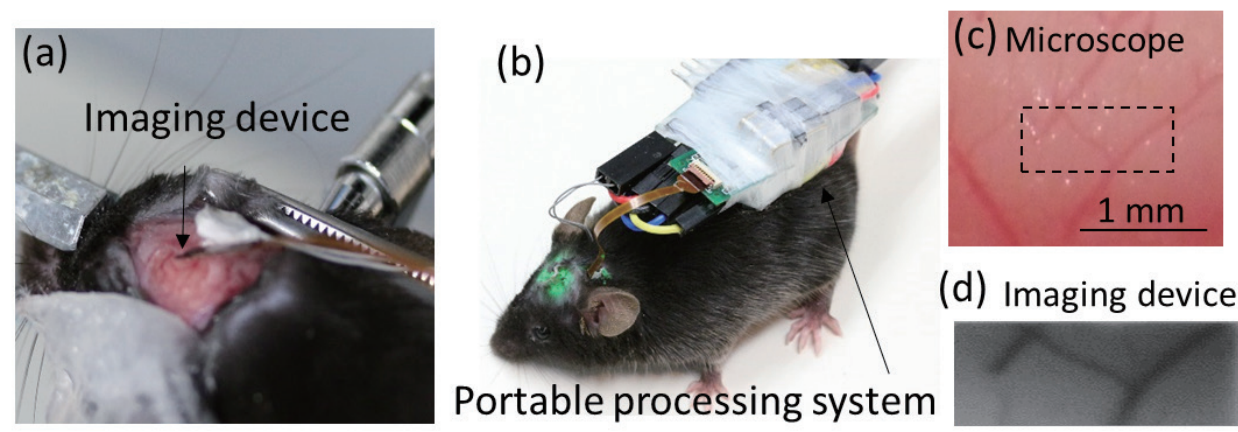

(d) Imaging device

Fig. 9. (Color online) Applying the imaging device with a portable processing system using a mouse. (a) Implantation of the imaging device in the mouse head. (b) A freely moving mouse shouldered the portable processing system. (c) Micrograph of the brain surface. (d) Image of the brain surface and blood vessels captured by the imaging device. 
Moreover, we deliberated on the exposure time of our imaging device for fluorescence imaging with DAF-FM DA. Because the response time of DAF-FM DA is fast and its fluorescence intensity gradually increases when reacting with NO within HUVECs, ${ }^{(15)}$ a $25 \mathrm{~ms}$ exposure time is suitable for the fluorescence detection of DAF-FM DA, as shown Fig. 8(a).

Finally, we demonstrated that our system can be applied to imaging applications with a freely moving animal by implanting our device and capturing the mouse brain surface. As shown in Figs. 9(c) and 9(d), the image of the brain tissue and blood vessels captured by our imaging device is clear and is the same as that captured by a microscope. Moreover, our previous research ${ }^{(16)}$ contributed to the experiments using small animals by demonstrating that the imaging device is less invasive and can be used in a long-term experiment for effectively observing animal behavior.

\section{Conclusions}

In this paper, we presented our developed small imaging device with a portable processing system for fluorescence detection in living cells. We demonstrated that our device is biocompatible and suitable for cell cultures. Furthermore, we reported our achievement in detecting green fluorescent images of DAF-FM DA by using our portable system. Finally, we demonstrated that our device can capture images of the brain tissue in a freely moving mouse. These results strongly suggest that our system is a powerful tool for fluorescence imaging not only in living cells, but also in freely moving animals.

\section{Acknowledgements}

This work was supported by the Japan Society for the Promotion of Science Grant-in-Aid for Scientific Research (A), \#26249051 and the VLSI Design and Education Center (VDEC), the University of Tokyo, in collaboration with Cadence Corporation and Mentor Graphics Corporation.

\section{References}

1 R. SoRelle: Circulation 98 (1998) 2365.

2 N. Omer, A. Rohilla, S. Rohilla, and A. Kushnoor: Int. J. Pharm. Sci. Drug Res. 4 (2012) 105.

3 J. Kim, M. Montagnani, K. K. Koh, and M. J. Quon: Circulation 113 (2006) 1888.

4 M. Hermann, A. Flamme, and T. F. Lüscher: J. Clin. Hypertens. S12 (2006) 17.

5 A. Peliowski: Paediatr. Child Health 17 (2012) 95.

6 N. A. Terpolilli, S. Kim, and S. C. Thal: Circ. Res. 110 (2012) 727.

7 Y. Y. Woldmana, J. Sunb, J. L. Zweierb, and V. V. Khramtsovb: Free Radic. Bio. Med. 47 (2009) 1339.

8 N. Hogg: Free Radic. Bio. Med. 49 (2010) 122.

9 E. L. Heinzen and G. M. Pollack: J. Pharmacol. Toxicol. Methods 48 (2002) 139.

10 X. Shia, T. Renb, and A. L. Nuttall: Hearing Res. 172 (2002) 73.

11 Y. Sunaga, H. Yamaura, M. Haruta, T. Yamaguchi, M. Motoyama, Y. Ohta, H. Takehara, T. Noda, K. Sasagawa, T. Tokuda, Y. Yoshimura, and J. Ohta: Jpn. J. Appl. Phys. 55 (2016) 03DF02.

12 S. Kima, D. Leea, I. Kima, D. Sohna, J. Parkb, B. Choib, and S. Kima: Kaohsiung J. Med. Sci. 28 (2012) 123.

13 B. E. Saleh and M. C. Teich: Fundamentals of Photonics, ed. J. W. Goodman (J Wiley, Hoboken, 1991) Chap. 17.

14 J. B. Pawley: Handbook of Biological Confocal Microscopy (Springer Science Business Media, New York, 2006) 3rd ed., Chap. 2.

15 M. Maiwulanjiang, C. W. C. Bi, P. S. C. Lee, G. Xin, A. Miernisha, K. M. Lau, A. Xiong, N. Li, T. T. X. Dong, H. A. Aisa, and K. W. K. Tsim: PLoS ONE 10 (2015) e0116761.

16 M. Haruta, C. Kitsumoto, T. Yamaguchi, H. Takehara, T. Noda, K. Sasagawa, T. Tokuda, and J. Ohta: Jpn. J. Appl. Phys. 53 (2014) 04EL05. 
About the Authors
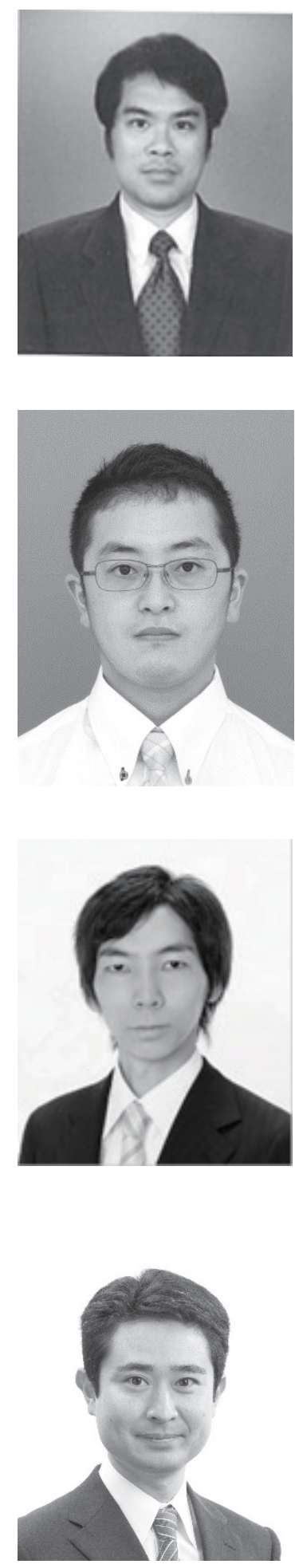

Anek Wuthayavanich received both his B.E. and M.E. in Electrical Engineering degrees from Chulalongkorn University, Bangkok, Thailand, in 2004 and 2007, respectively. Since 2014, he has been a Ph.D. student in Materials Science at the Photonic Device Science Laboratory, Nara Institute of Science and Technology (NAIST), Nara, Japan. His research focuses on the application of image sensors for the detection of biological signals.

Makito Haruta received his B.E. in Bioscience and Biotechnology degree from Okayama University, Okayama, Japan in 2009, M.S. in Biological Science degree from Nara Institute of Science and Technology (NAIST), Nara, Japan in 2011, and Dr. Eng. in Material Science degree from NAIST in 2014. In 2014, he joined the Graduate School of Materials Science, NAIST, as a postdoctoral fellow. His research interest is on brain imaging devices for understanding brain functions related to animal behaviors.

Hiroaki Takehara received his B.S. in Materials Engineering degree and M.S. and Ph.D. (Eng.) in Bioengineering degrees from the University of Tokyo, Japan, in 2008, 2010, and 2013, respectively. He was a research fellow of the Japan Society for the Promotion of Science from 2010 to 2013 and a postdoctoral fellow at Tokyo Women's Medical University from 2013 to 2014. From 2014, he has been an assistant professor at Nara Institute of Science and Technology (NAIST). His research interests include microfabrication technology, bio-MEMS devices, and implantable devices for life science and biomedical applications.

Toshihiko Noda received his B.E. and M.E. in Electrical and Electronic Engineering degrees in 2001 and 2003, respectively, and Ph.D. in Engineering degree in 2006, all from Toyohashi University of Technology (TUT), Aichi, Japan. He was a postdoctoral researcher of Venture Business Laboratory in TUT from 2006 to 2007. He joined the faculty of the Intelligent Sensing System Research Center in TUT from 2008 as assistant professor. Since 2009, he has been an assistant professor in Nara Institute of Science and Technology (NAIST), Nara, Japan. His current research interests focus on retinal prosthesis devices and bio-imaging with CMOS image sensors. 

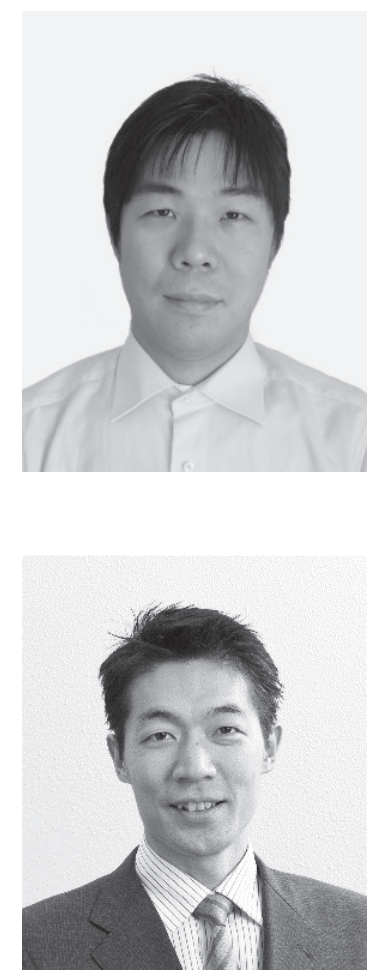

Takashi Tokuda received his B.E. and M.E. in Electronic Engineering degrees from Kyoto University, Kyoto, Japan, in 1993 and 1995, respectively. He received his Ph.D. in Materials Engineering degree from Kyoto University in 1998. He had been an assistant professor since 1999 and has been working as an associate professor since 2008 at the Graduate School of Materials Science, Nara Institute of Science and Technology (NAIST). His research interests include CMOS image sensors, retinal prosthesis devices, bioimaging sensors, and biosensing devices.

Jun Ohta received his B.E., M.E., and Dr. Eng. in Applied Physics

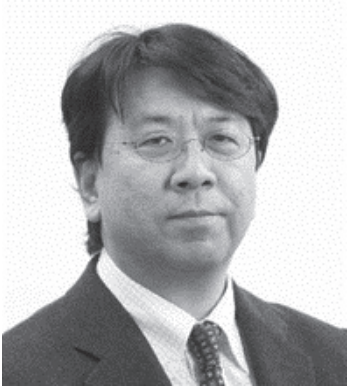
degrees, all from the University of Tokyo, Japan, in 1981, 1983, and 1992, respectively. In 1983, he joined Mitsubishi Electric Corporation, Hyogo, Japan. From 1992 to 1993, he was a visiting scientist at the Optoelectronics Computing Systems Center, University of Colorado at Boulder. In 1998, he joined the Graduate School of Materials Science, Nara Institute of Science and Technology (NAIST), Nara, Japan as Associate Professor. He was appointed as Professor in 2004. His current research interests are smart CMOS image sensors for biomedical applications and retinal prosthetic devices. He serves as an Associate Editor of IEEE Sensors Journal and an Editorial Board member of the Journal of Engineering, IET. He is a Fellow of the Japan Society of Applied Physics and the Institute of Image, Information, and Television Engineers, and a Senior Member of IEEE. 